\title{
Petrografía, propiedades físicas y durabilidad de algunas rocas utilizadas en el patrimonio monumental de Catalunya, España
}

\author{
ROSA M. ESBERT (*); ROSA M. MARCOS (**); JORGE ORDAZ (*); \\ MODESTO MONTOTO $\left(^{\star}\right)$; LUIS M. SUAREZ DEL RIO $\left({ }^{*}\right)$; VICENTE G. RUIZ DE ARGANDOÑA $(*)$; \\ LOPE CALLEJA (*); F. JAVIER ALONSO (*) y ANGEL RODRIGUEZ-REY (*) \\ Fecha de recepción: 14-V1-1989
}

\section{RESUMEN}

Se realiza la descripción petrográfica y se determina una serie de propiedades físicas de algunas rocas utilizadas en el patrimonio arquitectónico de Catalunya conocidas, en el ámbito de las "rocas de construcción", con los nombres de piedras de Girona. Folgueroles y Vinaixa. Mediante ensayos de envejecimiento artificial acelerado, se hacen predicciones sobre la durabilidad de las mismas.

\section{SUMMARY}

The petrographical description and the values of some physical properties of three rocks used in the architectonic patrimony of Catalunya are presented. These rocks are known, in the field of the "building stones" as Girona, Folgueroles and Vinaixa stones. By ageing tests, their durability is predicted.

\section{INTRODUCCION}

El diagnóstico de las diversas patologías que con frecuencia aparecen sobre las piedras monumentales, es un aspecto que resulta imprescindible conocer cuando se pretende realizar algún trabajo de conservación de las mismas.

Sin embargo, debe tenerse presente que en la génesis de estas patologías intervienen diversos factores, entre los cuales, los relacionados con las características petrofísicas de los materiales rocosos, así como su durabilidad, juegan un papel fundamental.

En este sentido, en el presente trabajo se documentan tres variedades rocosas de naturaleza sedimentaria, ampliamente utilizadas en el patrimonio monumental de Catalunya: las piedras de Girona, Folgueroles y Vinaixa.

La piedra de Girona es una caliza nummulítica de edad miocénica que procede de canteras ubicadas en la provincia de Gerona, muchas de ellas abandonadas en la actualidad. Se ha utilizado en la construcción de la Catedral de Girona, Monasterio de Sant Pere de Galligans, etc.

(*) Dr. en Geología Dpto. de Geología. Univ. de Oviedo.

$\left.{ }^{* \star}\right)$ Licenciada en Geología Dpto. de Geología. Univ. de Oviedo.
La piedra de Folgueroles es una arenisca de edad eocénica procedente de la provincia de Barcelona, y más concretamente de la comarca de Osona. Ha sido muy utilizada en la zona de Vic (Barcelona) (Seminario y Catedral) asi como en algunas construcciones de Barcelona capital (Monumento a Mossèn Cinto Verdaguer, etc).

La piedra de Vinaixa o de Floreste procede de la provincia de Lérida, concretamente de la comarca de Les Garrigues. Es un material oligocénico que ha sido utilizado en la Seo Vella de Lleida, Monasterios de Poblet y Santes Creus, etc.

\section{NORMAS Y RECOMENDACIONES DE ENSAYO}

Las propiedades físicas se han determinado siguiendo las normas y recomendaciones siguientes:

Peso específico y porosidad: International Society for Rock Mechanics (I.S.R.M., 1981) (1).

Distribución porométrica: Técnicas de Porosimetría por inyección de mercurio (Alonso et al, 1987) (2).

Absorción de agua: Centro Nazionale de Ricer- 
ca - Istituto Centrale del Restauro (C.N.R.I.C.R., Normal 7/81) (3).

Desorción de agua: Alonso (1985) (4).

Succión capilar: C.N.R.-I.C.R., Normal 11/82 (5).

Permeabilidad al vapor de agua: C.N.R.-I.C.R., Normal 21/85 (6).

Resistencia a la compresión uniaxial y Módulo de elasticidad: I.S.R.M. (1981) (1).

Velocidad de propagación de ondas longitudinales: I.S.R.M. (1981) (1).

Los ensayos de envejecimiento artificial acelerado se han llevado a cabo según las recomendaciones de la R.I.L.E.M. (1980) (7) (Reunión Internacional de Laboratorios de Ensayos de Materiales).

\section{RESULTADOS OBTENIDOS}

\subsection{Caliza de Girona}

\section{CARACTERISTICAS PETROGRAFICAS}

\section{Petrografía}

Caliza nummulítica de color gris y aspecto masivo microcristalino, en la que destaca la presencia de fósiles (nummulites).

La roca es compacta, coherente y relativamente homogénea (Fig. 1).

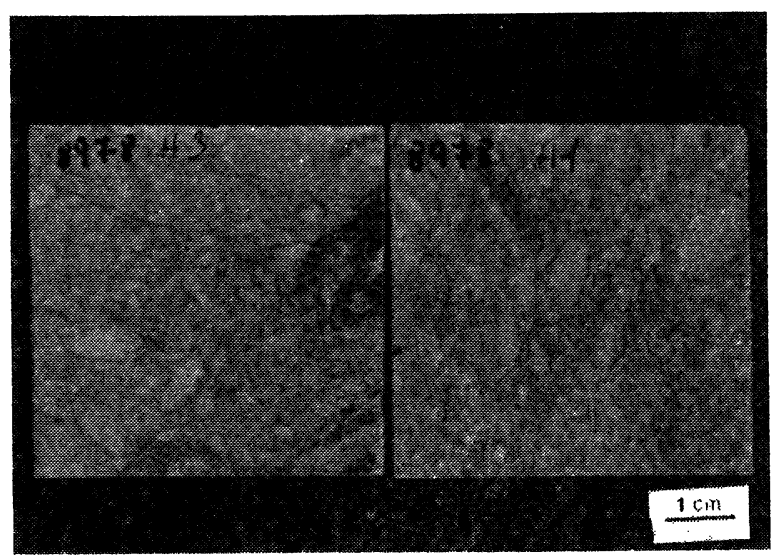

Fig. 1.-Aspecto macroscópico de la caliza de Girona. Pueden observarse los fósiles (nummulites) de color claro: $y$, con disposición intersticial y color más oscuro, la matriz arenosa.
El componente esencial es la calcita, presentando también algo de dolomita. Entre los minerales terrígenos se observan granos de feldespatos, de cuarzo y, en proporción mucho menor, minerales arcillosos y óxidos de hierro.

La textura es clástica, granosoportada, destacando los fósiles de gran tamaño (nummulites). Intersticialmente aparece una matriz arenosa con presencia de micrita y, en algunas zonas, cemento espático (Fig. 2).

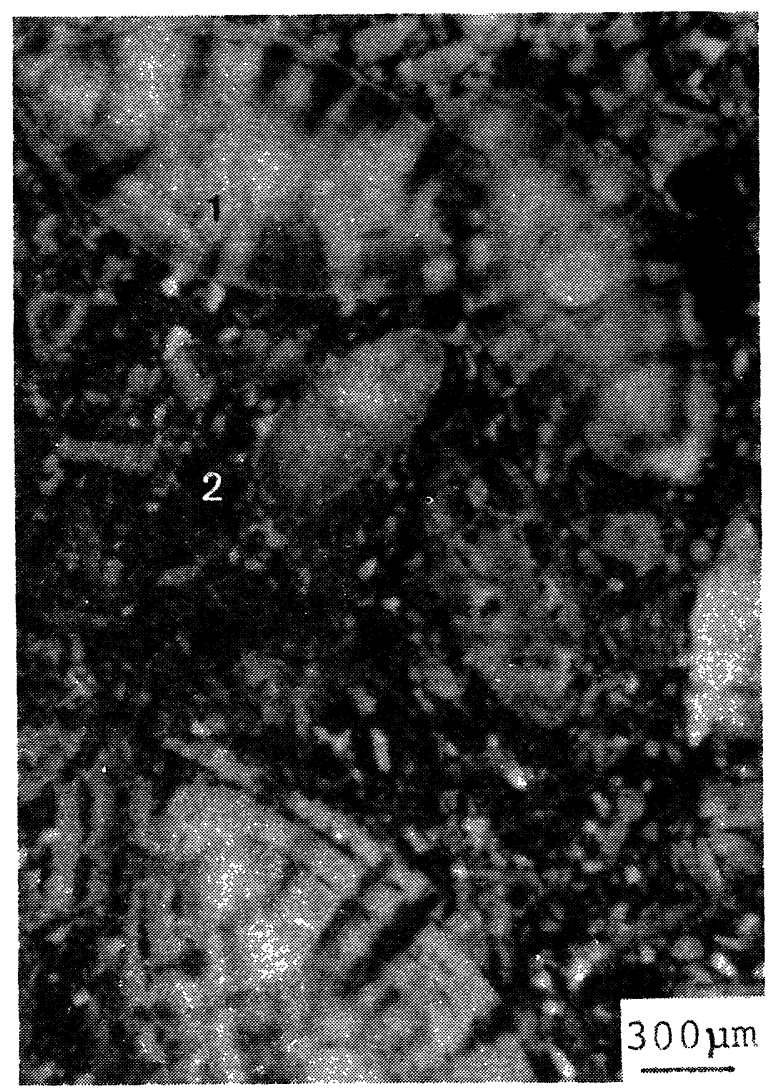

Fig. 2.-Textura de la caliza de Girona. Se observan nummulites interpenetrados (1) envueltos en una matriz arenosa (2). Microscopia óptica de polarización (M.O.P.), nicoles paralelos (N.P.).

Entre los componentes de mayor tamaño predominan los foraminiferos $y$, especialmente, los nummulites, situándose su tamaño medio alrededor de $2 \mathrm{~mm}$. Dichos fósiles están formados por calcita fibrosa, encontrándose las cámaras más internas cementadas por calcita espática $\mathrm{y}$, excepcionalmente, por dolomita.

Como fase de unión entre los foraminiferos se presenta una arena de grano fino, constituida por fragmentos de nummulites y otros restos fósiles, así como por granos de feldespatos y de cuarzo. El tamaño de estos granos puede situarse alrededor de $100 \mu \mathrm{m}$. Intersticial- 
mente los granos más pequeños presentan como fase de unión micrita $y$, localmente, cemento esparítico - a veces en continuidad óptica con los granos-, observándose de forma excepcional dolomita (Fig. 3).

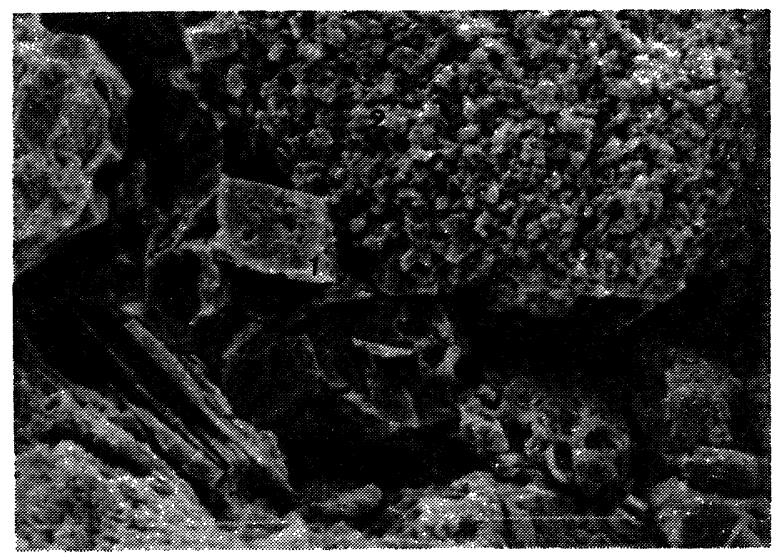

Fig. 3.-Detalle de la matriz de la caliza de Girona, mostrando el aspecto de los cristales rómbicos de dolomita (1), asi como un agregado de micrita (2). (M.E.B., 1 marcador $=10 \mu \mathrm{m})$.

Los foraminiferos suelen presentarse orientados, con un marcado grado de empaquetamiento. En algunas zonas se evidencian señales de disolución por presión, dando lugar a interpenetraciones de los componentes y al desarrollo de estilolitos más o menos marcados (Fig. 2).
La roca presenta muy baja proporción de espacios vacios, localizándose éstos, preferentemente, en la matriz arenosa y, en particular, en la fracción micrítica.

\section{Análisis difractométrico}

La calcita es el componente fundamental identificándose también dolomita, cuarzo y feldespato.

Los porcentajes de dichos componentes, dentro de la fracción superior a $2 \mu \mathrm{m}$, son los siguientes:

$\begin{array}{lr}\text { calcita } & 82,9 \% \\ \text { dolomita } & 2,4 \% \\ \text { feldespato } & 9,6 \% \\ \text { cuarzo } & 5,1 \%\end{array}$

\section{PROPIEDADES FISICAS}

En la Tabla I se presentan los valores de propiedades físicomecánicas obtenidos para este material. Aunque dichos valores son suficientemente elocuentes, se ha considerado oportuno hacer una serie de comentarios respecto a dichas propiedades, al igual que en el resto de los materiales estudiados.

La porosidad abierta de esta piedra es muy baja para el tipo de litología considerado.

TABLA 1

Propiedades físicas de las rocas estudiadas

\begin{tabular}{|l|c|c|c|}
\cline { 2 - 4 } \multicolumn{1}{c|}{} & Girona & Folgueroles & Vinaixa \\
\hline CM & HUE7,5YRN6/gray & HUE5Y6/1 gray & HUE10YR7/1-7/2 light gray \\
$\rho \mathrm{d}$ & 2.670 & 2.460 & 2.250 \\
$\mathrm{n}_{0}$ & 0,5 & 7,1 & 21,3 \\
Wa & $0,37 \pm 0,04$ & $2,47 \pm 0,04$ & $5,36 \pm 0,44$ \\
Wd & $0,11 \pm 0,02$ & $0,09 \pm 0,02$ & $0,05 \pm 0,04$ \\
$\mathrm{C}$ & $0,30 \pm 0,03$ & $7,80 \pm 0,01$ & $55,5 \pm 0,02$ \\
Kv & 0,180 & 0,314 & 0,434 \\
Rc & $165 \pm 15$ & $70 \pm 10$ & $58 \pm 5$ \\
E & $6,7 \times 10^{4}$ & $1,9 \times 10^{4}$ & $2,0 \times 10^{4}$ \\
U.M.M. & 85 & 60 & 75 \\
Vp & $5.695 \pm 100$ & $3.180 \pm 50$ & $3.825 \pm 75$ \\
\hline
\end{tabular}

$\mathrm{CM}$ : Color (Munsell); $\rho \mathrm{d}$ : peso especifico $\left(\mathrm{Kg} / \mathrm{m}^{3}\right) ; \mathrm{n}_{0}$ : porosidad abierta (\%); Wa: absorción de agua en equilibrio (\%); Wd: desorción de agua en equilibrio (\%); C: coeficiente de absorción capilar $\left(\mathrm{kg} / \mathrm{m}^{2} \cdot \mathrm{h}^{1 / 2}\right) ; \mathrm{Kv}$ : permeabilidad al vapor de agua $\left(\mathrm{g} / \mathrm{m}^{2} \cdot 24 \mathrm{~h}\right)$; Rc: resistencia a la compresión uniaxial (MPa); E: módulo de elasticidad tangente (MPa); U.M.M.: umbral de microfisuración mecánico (\% Rc); Vp: velocidad de ondas longitudinales (m/s). 
Como consecuencia de la baja porosidad de esta roca $(0,5 \%)$, la curva de distribución porométrica es poco precisa; no obstante, pueden señalarse dos tendencias en los tamaños de poro, una alrededor de 0,01 $\mu \mathrm{m}$, y otra alrededor de $5 \mu \mathrm{m}$. (Fig. 4).

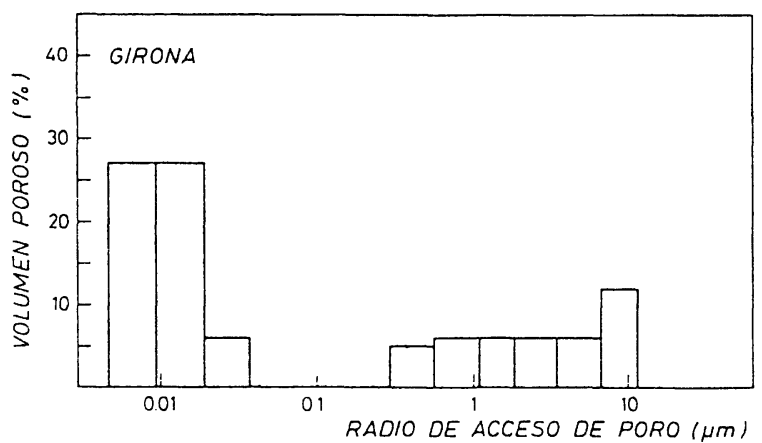

Fig. 4.-Distribución de tamaños de poro, obtenida mediante porosimetría por inyección de mercurio.

La capacidad de absorción de agua de esta piedra es baja. La Fig. 5 representa la variación del grado de saturación $\left(\mathrm{S}_{\mathrm{s}}\right.$ ) con respecto al tiempo. En ella puede verse cómo la absorción es relativamente lenta, alcanzándose aproximadamente el $50 \%$ de agua absorbida a las 16 horas de comenzado el ensayo, y el $90 \%$ a las 144 horas.

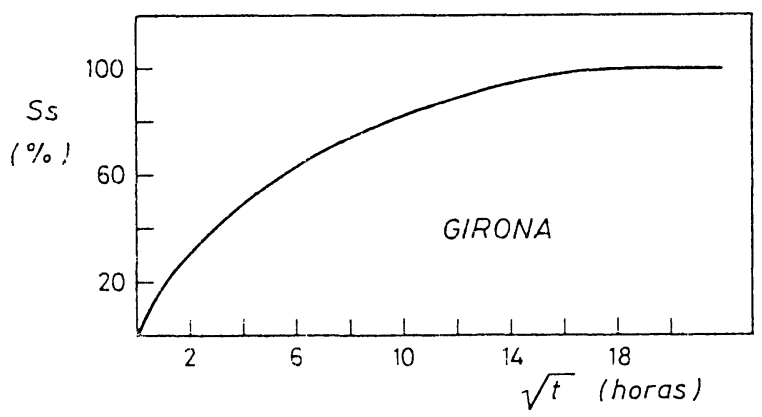

Fig. 5.-Absorción libre de agua: Grado de saturación (Ss) en función del tiempo $(\sqrt{t})$.

En la Fig. 6 se representa la cinética de evaporación de agua, pudiendo observarse que es necesario llegar a períodos de tiempo relativamente altos (360 huras) para conseguir el máximo secado en las condiciones ambientales, lo cual indica que, en desorción libre, este material presenta una moderada retención de agua.

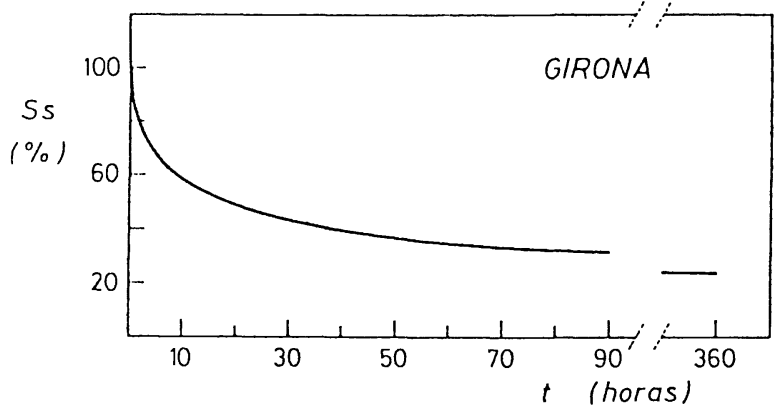

Fig. 6.-Desorción de agua: Grado de saturación (Ss) en función del tiempo (t).

El coeficiente de capilaridad puede considerarse muy bajo para este tipo de piedra. En la Fig. 7 puede verse la variación del incremento de masa por unidad de superficie en función del tiempo, manifestándose un comportamiento de "absorción lenta", y manteniéndose el mismo durante todo el ensayo.

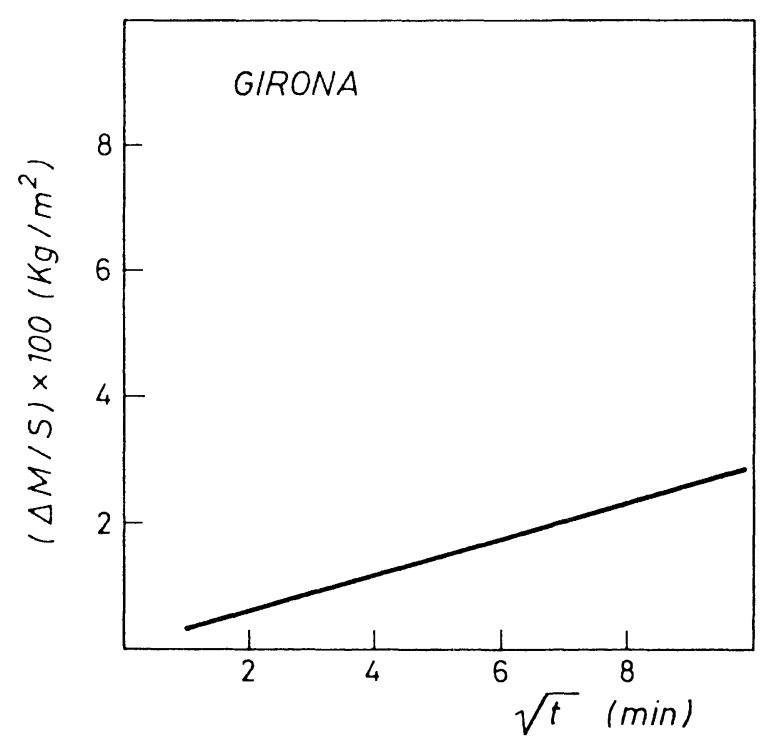

Fig. 7.-Absorción capilar: Incremento de peso por unidad de superficie $(\Delta M / S)$ en función del tiempo $(\sqrt{t})$.

El valor de la permeabilidad al vapor de agua en esta piedra es el más bajo de entre las variaciones estudiadas.

Las características mecánicas determinadas (Rc y E) sitúan a la piedra de Girona, según la clasificación de Deere y Miller (1966) (8), en el grupo BM (resistencia alta y módulo relativo medio). La curva esfuerzo-deformación es elasto-plástica (Fig. 8), estando el tramo de deformación plástica restringido a la última parte 
de la curva (correspondiente al tramo inelástico previo a la fractura). El Umbral de Microfisuración Mecánica, U.M.M., (es decir, la presión a la cual se inicia el desarrollo y/o creación de nuevas microfisuras) expresado como un porcentaje de la resistencia a la compresión y establecido mediante el registro de la emisión acústica, se localiza también a niveles altos de resistencia ( $85 \%$ de Rc). El tipo de fractura final fue por partición vertical.

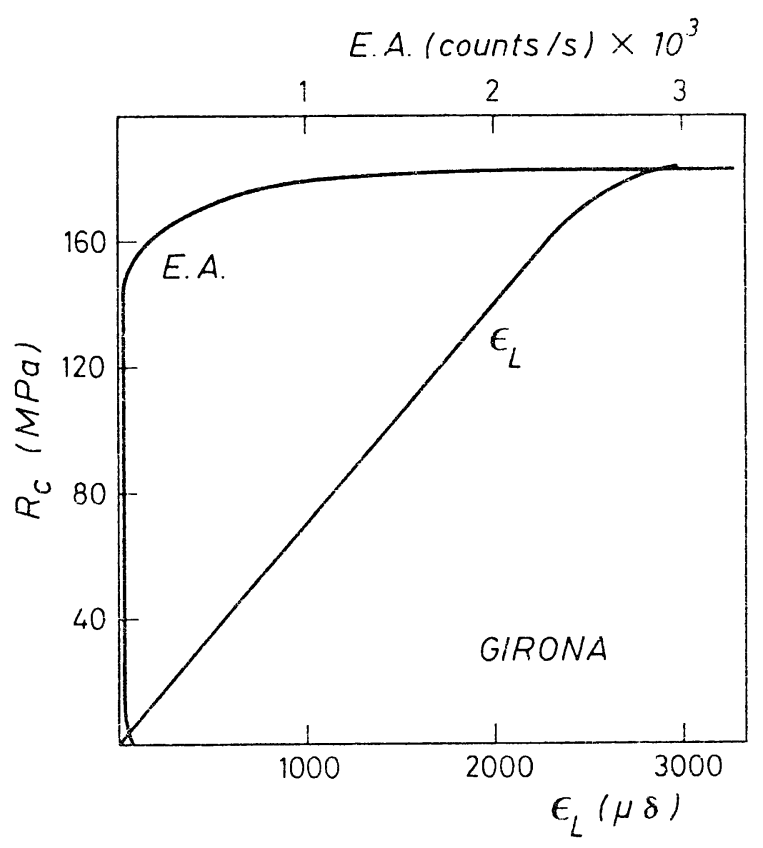

Fig. 8.-Curva esfuerzo-deformación con emisión acústica.

\section{ENSAYOS DE DURABILIDAD}

\section{Heladicidad}

La pérdida de masa experimentada por esta piedra en este ensayo es irrelevante $(0,017 \%$ después de los 30 ciclos).

El aspecto visual que presenta al final del ensayo es semejante al que presentaba al inicio del mismo; es decir, no se aprecia deterioro externo alguno.

\section{Cristalización de sales}

La pérdida de masa, con relación al peso inicial de las muestras, es insignificante $(0,093 \%$ después de los 10 ciclos).

La alteración superficial es inapreciable a simple vista.

\subsection{Arenisca de Folgueroles}

\section{CARACTERISTICAS PETROGRAFICAS}

\section{Petrografía}

Arenisca feldespática de color gris verdoso y aspecto granudo difuso, con acusada heterometría granular. La roca es relativamente homogénea, compacta y coherente (Fig. 9).

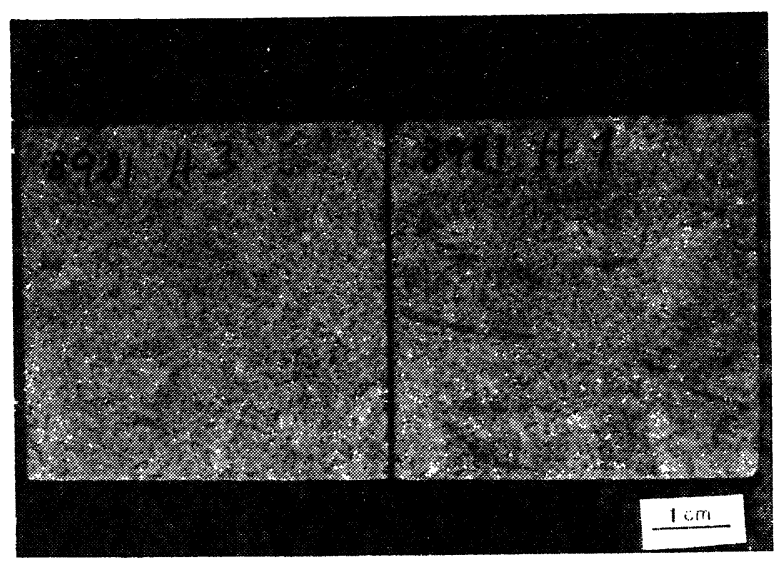

Fig. 9.-Aspecto macroscópico de la arenisca de Folgueoles. Puede observarse cierta heterometría en el tamaño de grano asi como la presencia de poros.

Los componentes esenciales son el cuarzo y también, con más bajo porcentaje, los feldespatos. Se observa también calcita y algo de dolomita. Destaca la presencia de clorita, identificándose algunos minerales arcillosos, fundamentalmente caolinita y algo de illita.

La textura es detrítica de grano medio, granosoportada, cementada mayoritariamente por calcita, ligeramente orientada y bien empaquetada.

Entre los granos de mayor tamaño $(>2 \mathrm{~mm})$ se pueden observar algunos fragmentos de granitos y de limolitas (Fig. 10). El tamaño medio de grano -que corresponde normalmente a cuarzos y feldespatos- se situa alrededor de $0,5 \mathrm{~mm}$ (Fig. 11). Entre ellos abundan los cuarzos policristalinos, observándose también, granos de chert.

Los feldespatos son mayoritariamente potásicos, bastante alterados, apareciendo, asimismo, algún grano de plagioclasa. También aparecen granos de carbonatos -a veces restos fósiles-y, en menor proporción, filosilicatos: moscovitas y cloritas. 


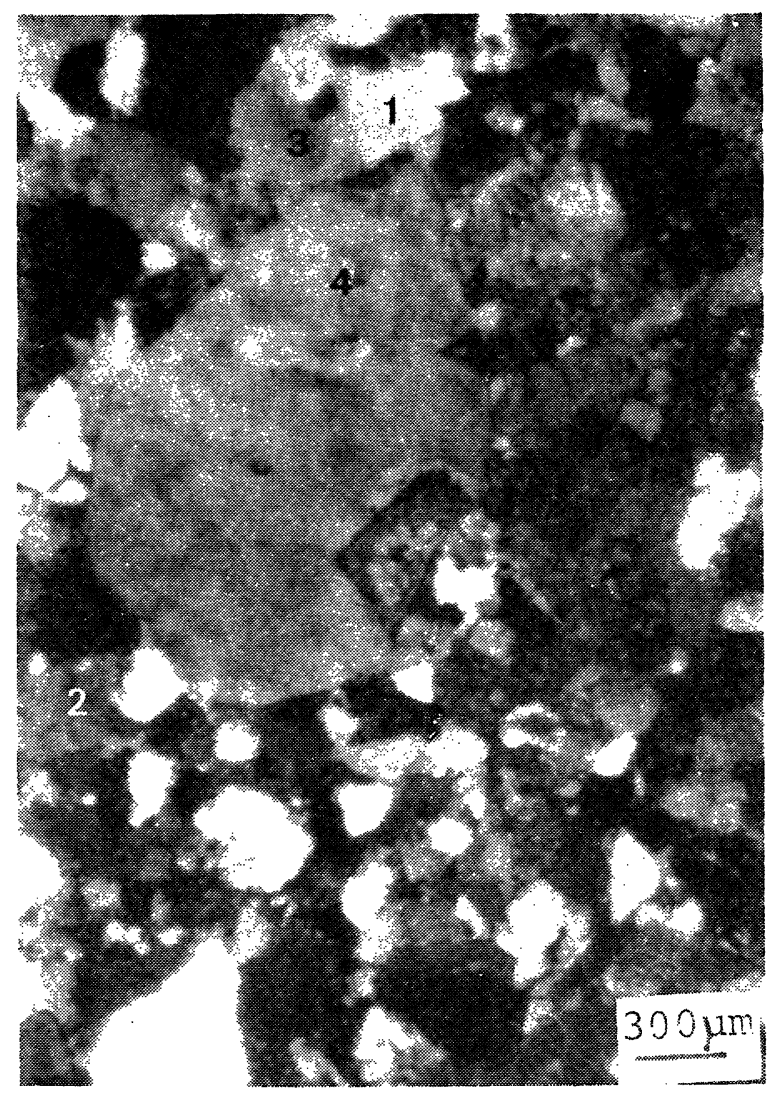

Fig. 10.-Textura de la arenisca de Folgueroles. Se aprecian granos de cuarzo (1), feldespatos (2) y calcita (3), asi como fragmentos líticos (4). (M.O.P., N.C.).

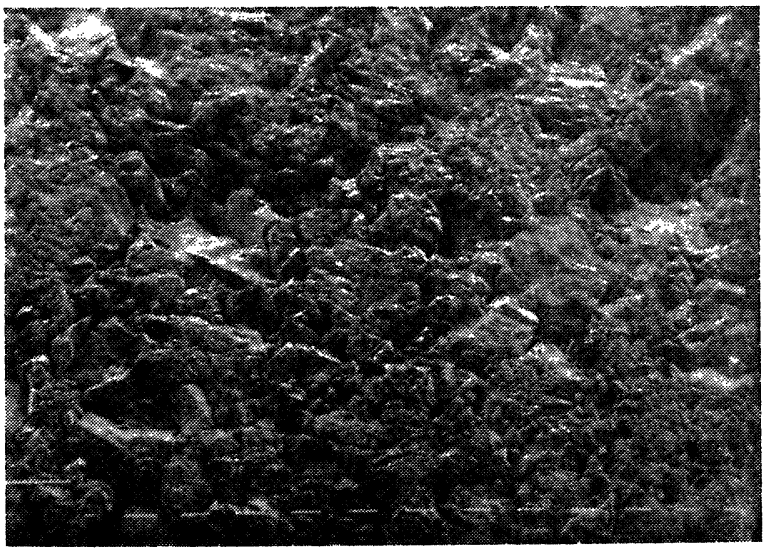

Fig. 11.-Textura de la arenisca de Folgueroles. (M.E.B., 1 marcador $=100 \mu \mathrm{m}$ ).

Como fase de unión entre los granos detríticos se encuentra frecuentemente cemento de calcita, con tamaño medio alrededor de las $100 \mu \mathrm{m}$. En algunas zonas también se observa clorita y minerales filosilicatados, mayoritariamente caolinita.

La roca presenta abundantes espacios vacios, localizados fundamentalmente en las zonas intergranulares y en relación con la fracción filosilicatada.

\section{Análisis difractométrico}

El cuarzo y los feldespatos constituyen los componentes fundamentales, identificándose también calcita, dolomita y minerales filosilicatos, fundamentalmente caolinita $y$, en menor proporción illita.

Los porcentajes de dichos componentes, dentro de la fracción superior a $2 \mu \mathrm{m}$, son los siguientes:

$\begin{array}{lr}\text { cuarzo } & 47,8 \% \\ \text { feldespatos } & 30,9 \% \\ \text { calcita } & 17,5 \% \\ \text { dolomita } & 3,8 \%\end{array}$

\section{PROPIEDADES FISICAS}

En la Tabla 1 se presentan los valores obtenidos para las propiedades calculadas.

La porosidad en esta piedra puede considerarse relativamente baja, para este tipo de materiales areniscosos.

El tamaño medio de radio de poro puede situarse alrededor de 0,06 $\mu \mathrm{m}$; es por tanto una roca esencialmente microporosa (rango intercuartílico: 0,03 a 0,15 $\mu \mathrm{m}$ ) (Fig. 12).

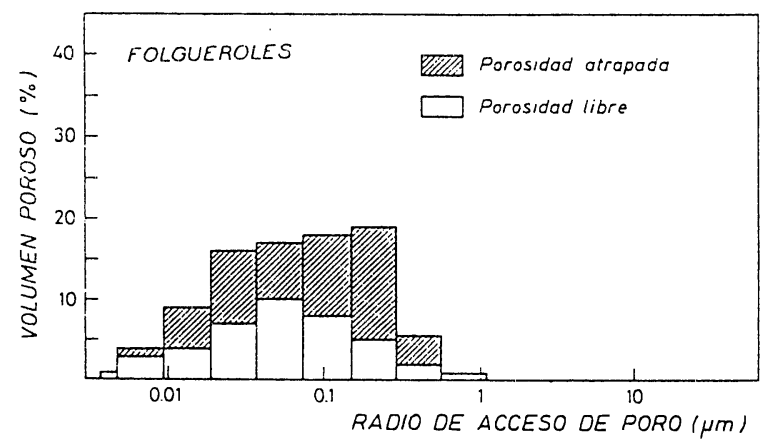

Fig. 12.-Distribución de tamaños de poro, obtenida mediante porosimetría por inyección de mercurio.

En la Fig. 13 se representa la curva de variación del grado de saturación $\left(\mathrm{S}_{\mathrm{s}}\right)$ con respecto al tiempo. En ella puede observarse que la absorción es muy rápida, alcanzándose aproximadamente el $90 \%$ de agua absorbida a las 4 horas de comenzado el ensayo para, poste-

MATERIALES DE CONSTRUCCION, Vol. 39, n.214, abril/mayo/junio 1989 
riormente, necesitar casi 121 horas para alcanzar el $100 \%$.

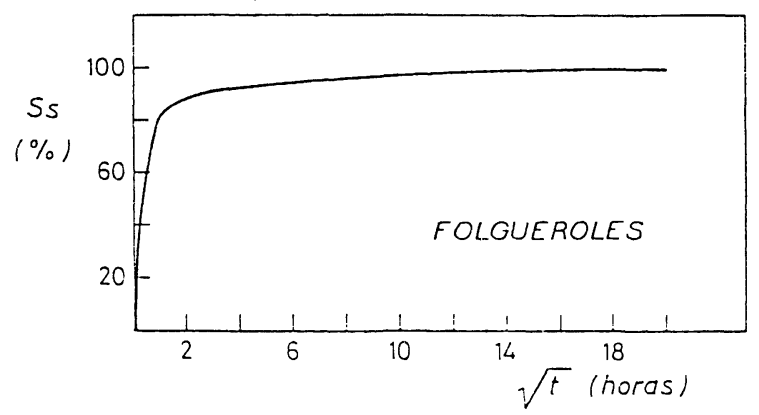

Fig. 13.-Absorción libre de agua: Grado de saturación (Ss) en función del tiempo $(\sqrt{t})$.

La cinética de desorción de agua está representada en la Fig. 14, pudiendo observarse que se necesita un período de tiempo relativamente corto (90 horas) para conseguir el secado en las condiciones ambientales; lo cual indica que, en desorción libre este material presenta muy poca retención de agua.

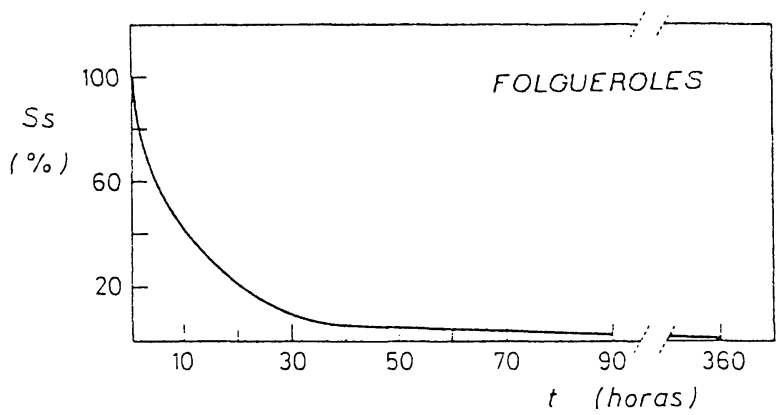

Fig. 14.-Desorción de agua: Grado de saturación (Ss) en función del tiempo (t).

El coeficiente de absorción por capilaridad es bajo. En la Fig. 15 puede verse la variación del incremento de masa por unidad de superficie en función del tiempo, manifestándose un comportamiento de "absorción muy rápida", y manteniéndose éste durante todo el ensayo.

El valor de la permeabilidad al vapor de agua obtenido para esta piedra es relativamente alto con respecto a los valores de otros materiales pétreos.

La resistencia a la compresión de la arenisca de Folgueroles puede considerarse media. Desde el punto de vista geomecánico, la roca pertenece al grupo $\mathrm{CL}$ (resistencia media y módulo relativo bajo). La curva esfuerzo-reformación (Fig. 16) es plasto-elasto-̣̂ástica, con un umbral de microfisuración mecánico que se sitúa en un $60 \%$ de la $R c$, y un registro de emisión acústica no muy elevado. La fractura final es de tipo conoclástico.

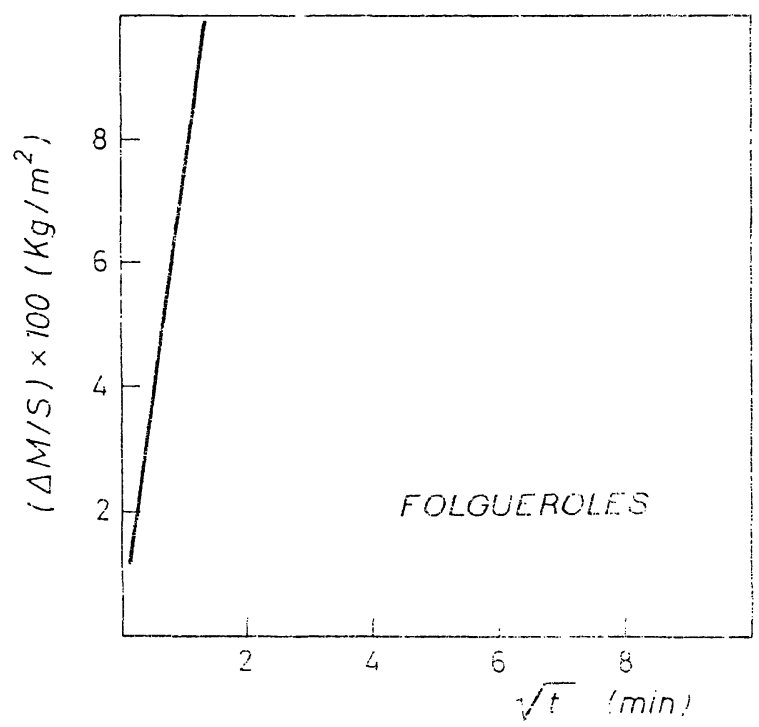

Fig. 15.-Absorción capilar: Incremento de peso por unidad de superficie $(\Delta M / S)$ en función del tiempo $(\sqrt{t})$.

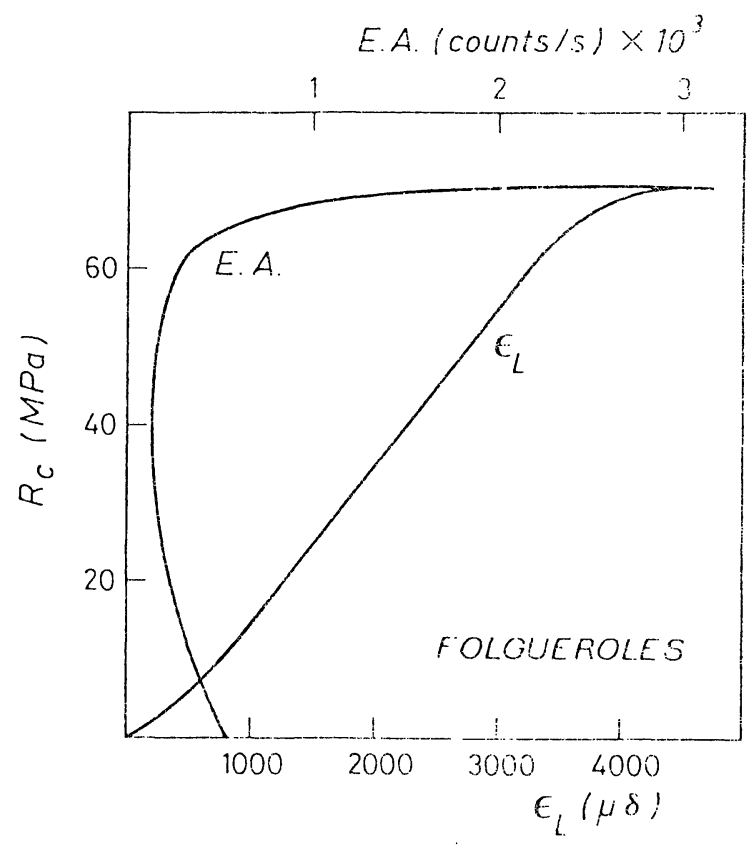

Fig. 16.-Curva esfuerzo-deformación con emisión acústica. 


\section{ENSAYOS DE DURABILIDAD}

\section{Heladicidad}

La pérdida de masa es poco significativa $(0,167 \%$ después de los 30 ciclos).

El aspecto externo de las probetas, una vez finalizado el ensayo, no se ha modificado.

\section{Cristalización de sales}

Las probetas comienzan a perder material, grano a grano, en los últimos ciclos. Hacia el final del ensayo se levantan pequeñas ampollas que llegan a romperse, de manera que la pérdida de material se acentúa. Sin embargo, la cantidad final de materia perdida es irrelevante $(1,2 \%$ después de los 10 ciclos).

Observadas, a simple vista, algunas superficies de las probetas resultan sensiblemente corroidas (Fig. 17),

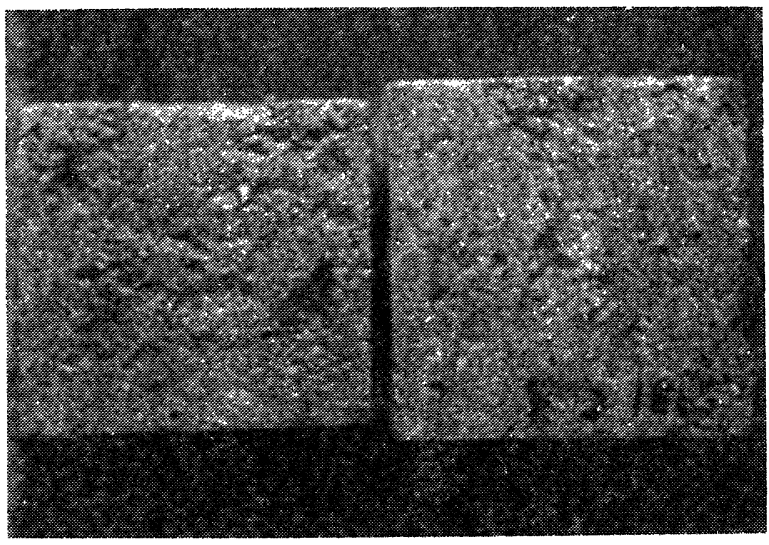

Fig. 17.-Aspecto de dos probetas de la piedra de Folgueroles transcurridos los diez ciclos de cristalización de sales, después de lavadas.

\subsection{Caliza de Vinaixa}

\section{CARACTERISTICAS PETROGRAFICAS}

\section{Petrografía}

Roca carbonatada, de color grisáceo y aspecto finamente granudo a cristalino. La roca es muy homogénea y coherente (Fig. 18).

Los componentes principales son la dolomita y la calcita. Presenta algunos granos de cuarzo, no observándose minerales arcillosos.

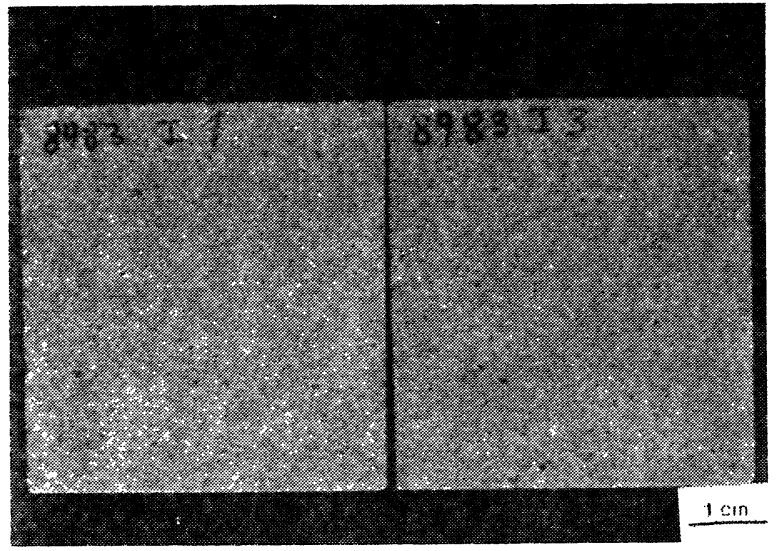

Fig. 18.-Aspecto macroscópico de la caliza de Vinaixa. Puede observarse la homogeneidad textural asi como la abundancia de poros.

La textura es de tipo granudo, detrítica, predominando los espacios vacios en las zonas intergranulares (Fig. 19).

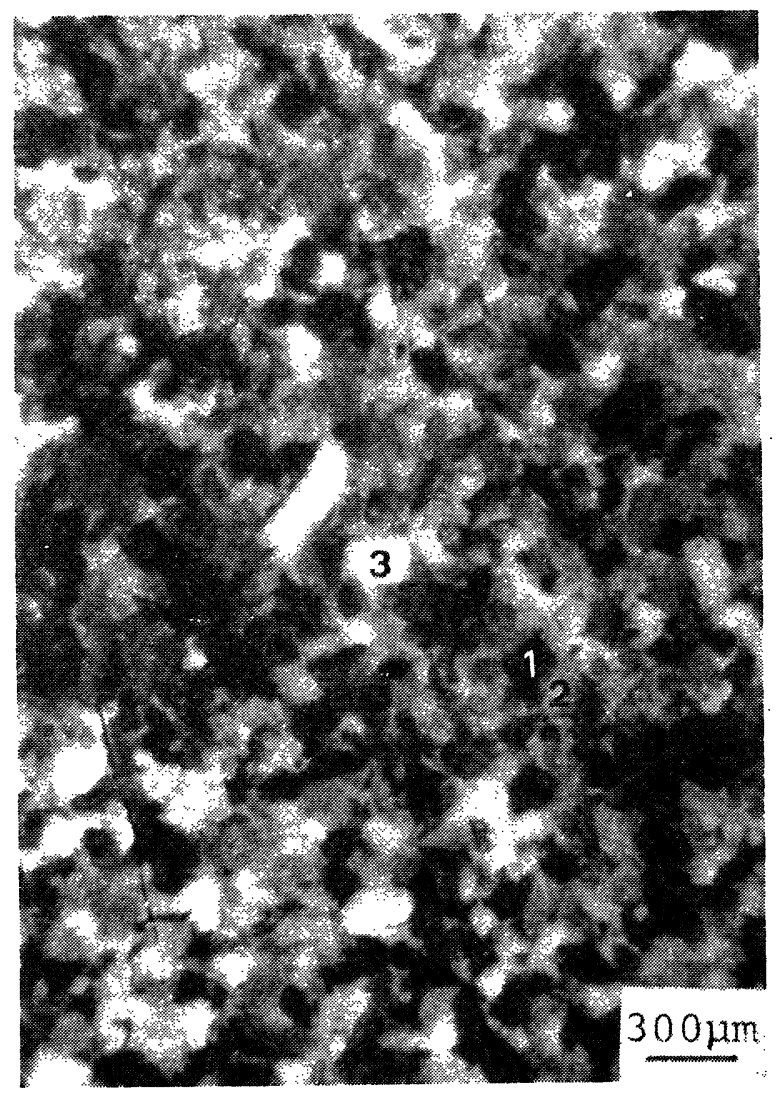

Fig. 19.-Textura de la caliza de Vinaixa. Destaca la presencia de granos micríticos (1) -oscuros-, espáticos (2) - más claros- y de cuarzo (3) - blancos-. (M.O.P., N.P.).

Los granos están formados por cristales únicos -espáticos-y agregados microcristalinos

MATERIALES DE CONSTRUCCION, Vol. 39, n.0214, abril/mayo/junio 1989 
- micríticos-, tanto de calcita como de dolomita, presentándose los distintos tipos de granos (esparita, micrita, doloesparita y dolomicroesparita) en parecidas proporciones; excepcionalmente se observan algunos fragmentos de fósiles.

Los granos muestran ligera heterometría, situándose su tamaño medio alrededor de $200 \mu \mathrm{m}$. La doloesparita presenta formas rómbicas más o menos redondeadas. Algunos granos de esparita poseen pequeñas orlas con cemento en continuidad óptica, formado por calcita ferrosa.

Normalmente los granos presentan contactos puntuales, predominando los espacios vacios en las zonas intergranulares. En dichas zonas, y de forma parcial, puede presentarse cemento carbonatado.

La roca posee abundantes espacios vacíos, de tamaño relativamente grandes, que se sitúan fundamentalmente en posiciones intergranulares, asi como en el interior de los granos micríticos y de los cristales de dolomita (Fig. 20).

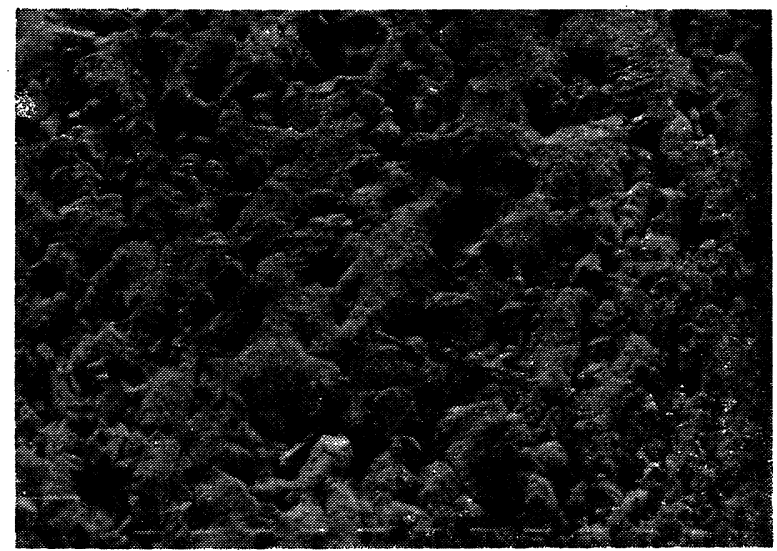

Fig. 20.-Caliza de Vinaixa: aspecto de la textura. Pueden observarse las morfologías de los distintos tipos de granos, así como el elevado número de espacios vacíos intergranulares. (M.E.B., 1 marcador $=100 \mu \mathrm{m}$ ).

\section{Análisis difractométrico}

Los minerales carbonatados, calcita y dolomita, constituyen los componentes fundamentales de la roca, identificándose también algo de cuarzo.

Los porcentajes de dichos componentes, dentro de la fracción superior a $2 \mu \mathrm{m}$, son los siguientes:

$\begin{array}{lr}\text { dolomita } & 50,9 \% \\ \text { calcita } & 40,3 \% \\ \text { cuarzo } & 8,9 \%\end{array}$

\section{PROPIEDADES FISICAS}

En la Tabla 1 se muestran los resultados de las propiedades físicas determinadas.

Esta piedra tiene un peso específico relativamente bajo en relación con su composición mineralógica, debido al alto valor de su porosidad.

La roca presenta una porosidad importante (21,3\%).

Los radios de acceso de poro son, para este tipo de piedra, relativamente grandes, pudiendo situarse su valor medio alrededor de $6 \mu \mathrm{m}$. Muestra baja dispersión de tamaños (rango intercuartílico: 2 a $10 \mu \mathrm{m}$ ) (Fig. 21).

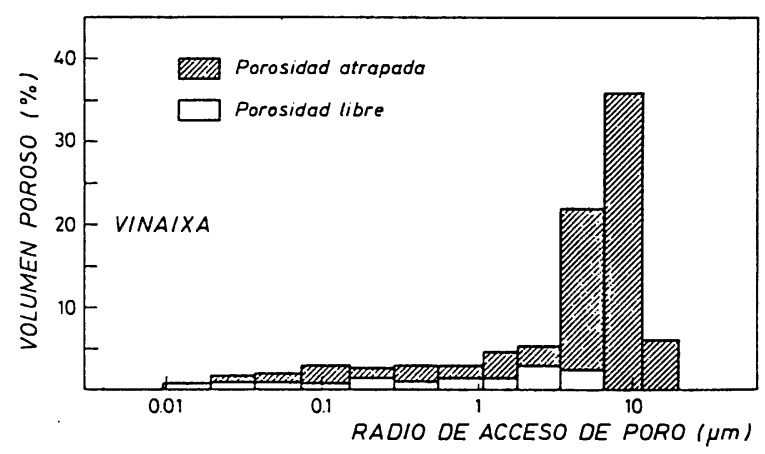

Fig. 21.-Distribución de tamaños de poro, obtenida mediante porosimetría por inyección de mercurio.

La capacidad de absorción de agua de esta variedad rocosa es alta. En la Fig. 22 se representa la variación del grado de saturación $\left(\mathrm{S}_{\mathrm{s}}\right)$ con respecto al tiempo. En ella puede verse cómo la absorción es rápida al principio, alcanzándose aproximadamente el $70 \%$ de agua absorbida en la primera hora después de comenzado el ensayo, para posteriormente hacerse más lenta y llegar al $98 \%$ a las 196 horas.

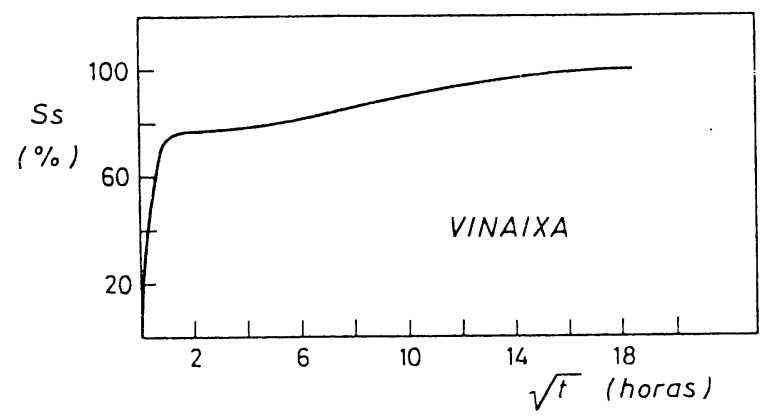

Fig. 22.-Absorción libre de agua: Grado de saturación (Ss) en función del tiempo libre $(\sqrt{t})$. 
En la Fig. 23 se representa la cinética de desorción de agua, pudiendo observarse que, en períodos de tiempo cortos, se alcanzan desorciones elevadas.

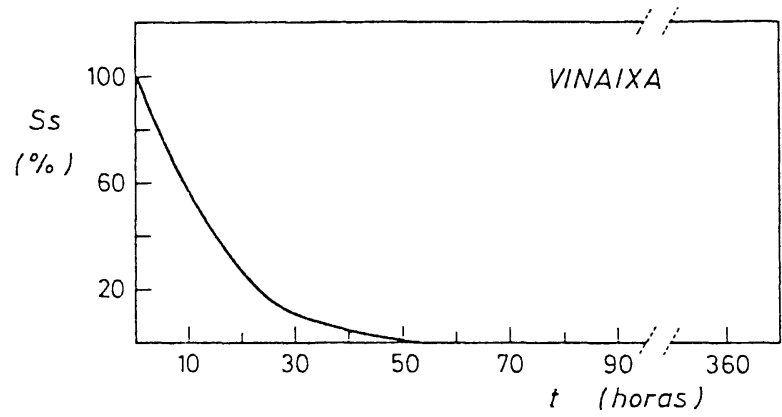

Fig. 23.-Desorción de agua: Grado de saturación (Ss) en función del tiempo (t).

El coeficiente de absorción capilar puede decirse que es relativamente alto para este tipo de materiales.

En la Fig. 24 puede verse la variación del incremento de masa por unidad de superficie en función del tiempo, manteniéndose el mismo durante todo el ensayo.

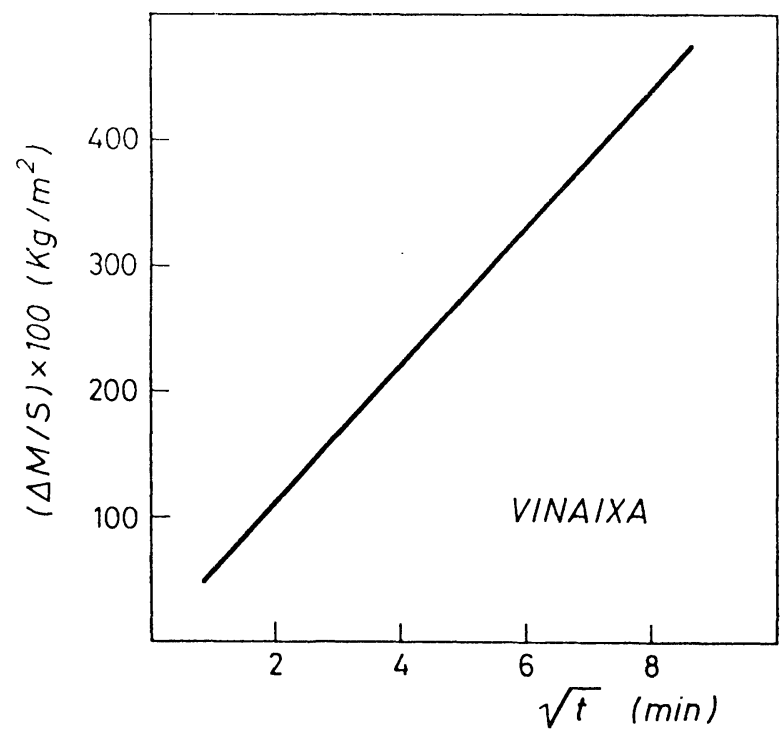

Fig. 24.-Absorción capilar: Incremento de peso por unidad de superficie $(\triangle M / S)$ en función del tiempo $(\sqrt{t})$.

El valor de la permeabilidad al vapor de agua es el más alto de los presentados por las variedades pétreas estudiadas.
La caliza de Vinaixa pertenece al grupo $\mathrm{CL}$ (resistencia media y módulo relativo bajo) dentro de la clasificación geomecánica de Deere y Miller, presentando un comportamiento deformacional plasto-elasto-plástico (Fig. 25). La fractura final se produce por cizalla, con disposición conoclástica. El U.M.M. se sitúa aproximadamente en el $75 \%$ de la Rc.

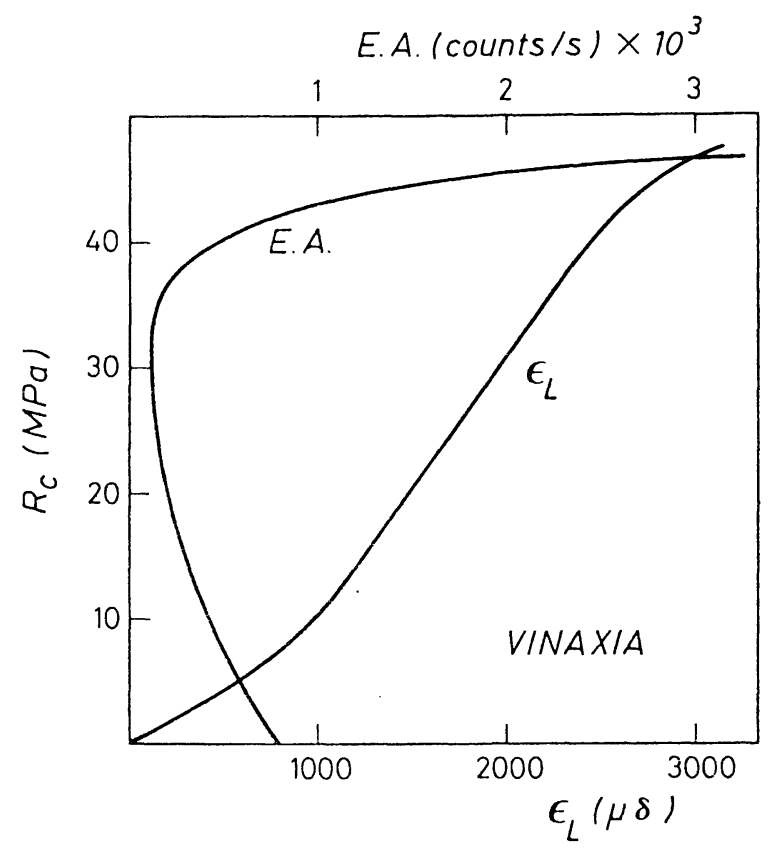

Fig. 25.-Curva esfuerzo-deformación con emisión acústica.

\section{ENSAYOS DE DURABILIDAD}

\section{Heladicidad}

La pérdida de masa en este ensayo es insignificante $(0,054 \%$ después de los 30 ciclos).

El deterioro superficial de las probetas ensayadas es inapreciable.

\section{Cristalización de sales}

La pérdida de masa medida tras los 10 ciclos realizados es insignificante $(1,68 \%)$.

El deterioro visual comienza y es mayor finalmente, en las aristas y vértices de las probetas, desagregándose grano a grano o en pequeñas desconchaduras (Fig. 26). 


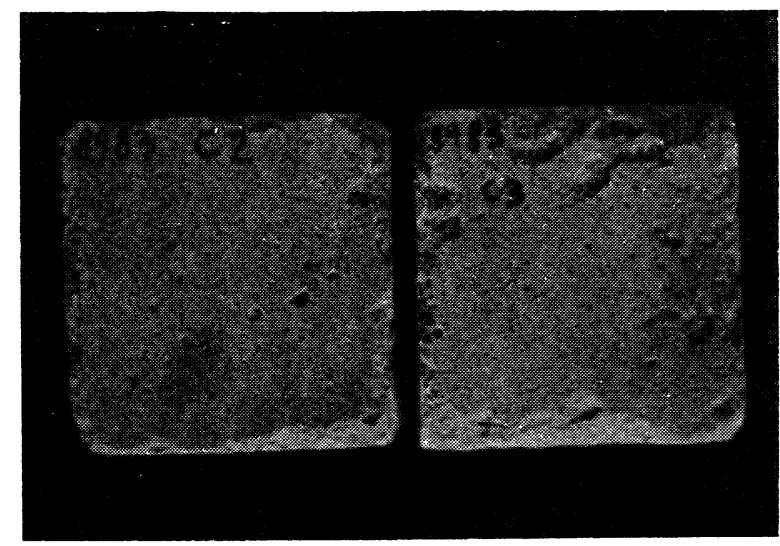

Fig. 26.-Probetas de piedra de Vinaixa después de los ciclos de cristalización de sales, tras ser lavadas.

\section{CONCLUSIONES}

Se ha llevado a cabo la caracterización petrográfica, física, mecánica y alterológica de los siguientes materiales pétreos utilizados en el Patrimonio monumental de Catalunya: caliza de Girona, arenisca de Folgueroles y caliza de Vinaixa.

La caliza de Girona corresponde a una caliza nummulítica, compacta y microcristalina. Su porosidad abierta es muy baja $(0,5 \%)$, lo que repercute en una escasa capacidad de absor- ción de agua, tanto por inmersión como por capilaridad, así como en una baja permeabilidad al vapor de agua. Su resistencia a la compresión uniaxial es elevada y su durabilidad las más alta de entre las estudiadas.

La piedra de Folgueroles es una arenisca feldespática, de textura detrítica y cementada mayoritariamente por calcita.

La porosidad abierta puede considerarse baja $(7,1 \%)$ para este tipo de materiales rocosos, siendo el tamaño medio de poro muy pequeño (radios de alrededor de 0,06 $\mu \mathrm{m}$ ). En consecuencia, la capacidad de absorción de agua es, en líneas generales, de moderada a baja. La presencia de determinados minerales filosilicatados (illita, caolinita) en la arenisca de Folgueroles parece no influir de modo significativo en su durabilidad, que puede considerarse relativamente alta.

La caliza de Vinaixa corresponde a una roca carbonatada, de textura granuda, detrítica, con abundantes espacios vacios intergranulares. La porosidad abierta para esta roca es elevada $(21,3 \%)$, con radios de poro medios de alrededor de $6 \mu \mathrm{m}$, lo que se traduce en una capacidad de absorción de agua relativamente alta, sobre todo por capilaridad. En cuanto a la resistencia mecánica y a la durabilidad, los valores obtenidos son inferiores a los de la caliza de Girona.

\section{AGRADECIMIENTOS}

Al Servicio de Patrimonio Arquitectónico de la Generalitat de Catalunya por el soporte económico de esta investigación.

Al Dr. Vicens Bonet i Ferrer, Catedrático de Materiales del Dpto. de Construcción de la Escuela Técnica Superior de Arquitectura de Barcelona, quien ha facilitado los datos referidos a las canteras y edad geológica de los materiales.

\section{BIBLIOGRAFIA}

(1) I.S.R.M. (1981): "Rock characterization testing and monitoring”. E.T. Brown (Ed.) Pergamon Press, Oxford, 211 pp.

(2) ALONSO, F. J.; R. M. ESBERT y J. ORDAZ (1987): "Caracterización del sistema poroso de calizas y dolomías". Bol. Geol. y Min. XLVIII-II, pp. 226-237.

(3) C.N.R.-I.C.R. (1981): "Assorbimento d'acqua per inmersione totale. Capacitá d'imbibizione". Normal 7/81, Roma, 5 pp.

(4) ALONSO, F. J. (1985): "Caracterización petrofísica y alterabilidad de calizas y dolomías". Tesis Doctoral. Facultad de Geologia. Universidad de Oviedo, $309 \mathrm{pp}$.

(5) C.N.R.-I.C.R. (1982): "Assorbimento d'acqua per capillaritá. Coefficiente di assorbimento capillare". Normal 11/82, Roma, $6 \mathrm{pp}$.

(6) C.N.R.-I.C.R. (1985): "Permeabilitá al vapor d'acqua". Normal 21/85, Roma, 5 pp.

(7) R.I.L.E.M (1980): "Essais recommandés pour mesurer l'altération des pierres et évaluer l'efficacité des métodes de traitement". Matériaux et Constructions, 13 (75), pp. 216-220.

(8) DEERE, D.U. y R.P. MILLER (1966): "Engineering classification and index properties for intact rock". Tech. Rept. N. ${ }^{\circ}$ AFWL-TR-65-116, Air Force Weapons Lab., Kirtland Air Force Base, Nuevo Méjico, U.S.A. 